\title{
Physics of coronal magnetic loops
}

\author{
Alexander V. Stepanov \\ Central Astronomical Observatory at Pulkovo, Russia, email: stepanov@gao.spb.ru
}

\begin{abstract}
Three approaches for coronal magnetic loops on the Sun and the stars are considered: (i) loop as MHD-resonator, (ii) loop as electric circuit, (iii) loop as a magnetic mirror trap.
\end{abstract}

\section{Introduction}

Space and VLBI observations gave us clear evidences that magnetic loops are the fundamental structures of coronae of the Sun and late type stars (e.g. Benz, Conway \& Güdel 1998; Schrjiver et al. 1999). Various energy release processes like flares, plasma heating, and charged particle acceleration take a place in coronal loops. Three approaches presented here serve to improve diagnostics of loop plasma parameters and to understand the physical processes in coronal loops.

\section{Loop as a resonator for MHD-waves}

More than three decades ago pulsations of solar radio bursts were associated with MHD oscillations of coronal magnetic loops (Rosenberg 1970). This idea was developed by many authors but only recently TRACE observations presented convincing evidence for the validity of this approach (Nakariakov et al. 1999). Fast sausage, slow MHD, Alfven, kink, and torsion modes of a coronal loop are used usually for the interpretation of the pulsations of solar emission (e.g. Aschwanden 2003). Here attention is paid that the ballooning mode of coronal loops is also important as an origin of solar and stellar pulsations.

As the first approximation the coronal magnetic loop can be investigated using the homogeneous plasma cylinder with radius $r$ and the length $l$ with fixed ends. Plasmas inside and outside the cylinder have density $\rho_{i}, \rho_{e}$, the temperature $T_{i}, T_{e}$, and the magnetic field $B_{i}, B_{e}$, correspondingly. Dispersion equation for eigen-oscillations of plasma cylinder can be written as (Meerson, Sasorov \& Stepanov 1978; Roberts, Edwin \& Benz 1984)

$$
\frac{J_{m}^{\prime}\left(\kappa_{i} r\right)}{J_{m}\left(\kappa_{i} r\right)}=\alpha \frac{H_{m}^{(1)^{\prime}}\left(\kappa_{e} r\right)}{H_{m}^{(1)}\left(\kappa_{e} r\right)}
$$

Here

$$
\kappa^{2}=\frac{\omega^{4}}{\omega^{2}\left(c_{s}^{2}+c_{A}^{2}\right)-k_{\|}^{2} c_{s}^{2} c_{A}^{2}}-k_{\|}^{2}, \quad \alpha=\frac{\kappa_{i} \rho_{i}}{\kappa_{e} \rho_{e}} \frac{\omega^{2}-k_{\|}^{2} c_{A i}^{2}}{\omega^{2}-k_{\|}^{2} c_{A e}^{2}},
$$

$c_{s}$ is the sound velocity, $c_{A}=B /(4 \pi \rho)^{1 / 2}$ is the Alfven velocity, $J_{m}$ and $H_{m}^{(1)}$ are the Bessel and Hankel functions of the first kind, $k_{\|}=s \pi / l, s=1,2,3, \ldots$ For slender $(r / l \ll 1)$ and dense $\left(\rho_{e} / \rho_{i} \ll 1\right)$ cylinder in the axial symmetrical case $(m=0)$ from Eq. (2.1) we obtain the frequency of fast magneto-sonic (fast sausage) oscillations, which 
is most effective in the context of modulation of the emission:

$$
\omega_{s}=\left(k_{\perp}^{2}+k_{\|}^{2}\right)^{1 / 2}\left(c_{s i}^{2}+c_{A i}^{2}\right)^{1 / 2} .
$$

The transverse wave number is $k_{\perp}=\lambda_{j} / r$, where $\lambda_{j}$ are the roots of the equation $J_{0}(\lambda)=0$. Loop oscillations undergo acoustic damping caused by the emission of waves into the surrounding medium. The damping rate is (Meerson, Sasorov \& Stepanov 1978)

$$
\gamma_{s}=\frac{\pi}{2} \omega_{s}\left(\frac{\rho_{e}}{\rho_{i}}-\frac{k_{\|}^{2}}{k_{\perp}^{2}}\right) .
$$

There is no damping for $\rho_{e} / \rho_{i}<k_{\|}^{2} / k_{\perp}^{2} \approx r^{2} / l^{2}$, e.g. for comparatively thick loop. In this case the total internal reflection takes place and plasma cylinder becomes an ideal resonator. As it follows from observations, the plasma density inside flare loops is two to three orders of magnitude larger than outside. So, there is a jump of impedance for fast magneto-sonic (FMS) waves. The acoustic damping of the FMS oscillations of coronal loops becomes insignificant and dissipative processes play main role. Under solar condition the most important damping mechanisms for FMS mode is the electron thermal conductivity (Stepanov et al. 2004) and the Q-factor of pulsations is:

$$
Q=\frac{\omega}{\gamma}=\frac{2 m_{e}}{m_{i}} \frac{P \nu_{e i}}{\beta^{2} \sin ^{2} 2 \theta} .
$$

Here $P=2 \pi / \omega, \beta=8 \pi p / B^{2}<1, \theta$ is the angle between the magnetic field $\mathbf{B}$ and wave vector $\mathbf{k}, \nu_{e i}$ is effective frequency of electron-ion collisions.

For ballooning mode the curvature of the loop magnetic field is important. Oscillations occur as the result of acting of two forces: the force dealing with gas pressure gradient and magnetic field line curvature $F_{1} \sim p / R$, and the back-ward force due to the magnetic field tension $F_{2} \sim B^{2} / R$. The period of the ballooning oscillations for typical flare loop $\left(l \sim 10^{10} \mathrm{~cm}, \beta \sim 0.1\right)$ can be write as (Stepanov et al. 2004)

$$
P_{b}=\frac{l}{c_{A}} \sqrt{\frac{1}{N^{2}-l \beta /(8 \pi r)}} \approx \frac{l}{c_{A} N} .
$$

Here $N$ is the number of plasma tongues in the loop, $l \approx \pi R$. Ideal MHD provides evidence for the analogy between the ballooning and FMS modes. So, to determine the Q-factor of ballooning mode one can use Eq. (2.4).

Both FMS and ballooning oscillations provide deep modulation of gyrosynchrotron emission of energetic electrons in a loop because they change the magnetic field, the scale of the emitting region, the plasma temperature, and density. Let us consider the effects of coronal loop oscillations on the flare microwave radiation observed by Nobeyama Radioheliograph $(\mathrm{NoRH})$ at $17 \mathrm{GHz}$ for which the optically thin gyrosynchrotron emission of nonthermal electrons is responsible (Stepanov et al. 2004). Follow to well-known Dulk's formulas the flux of optically thin gyrosynchrotron emission is $F \propto B^{\xi}$, where $\xi=$ $0.90 \delta-1.22, \delta$ is the spectral index of power-law electrons. Taking into account the conservation of the magnetic flux we can write the modulation depth as

$$
\Delta=\left(F_{\max }-F_{\min }\right) / F_{\max }=2 \xi \frac{\delta B}{B} .
$$

Here $\delta B$ is the deviation of loop magnetic field. Oscillations can excite due to the rapid enhancement of the gas pressure $\delta p \approx n \kappa_{B} T$ at the impulsive phase of solar flare. Hence we can get the following relation: $\beta \approx 2 \delta B / B=\Delta / \xi=\varepsilon$. From the formulas for periods, Q-factor (2.4) and modulation depth (2.6) we find the expressions for determination 
Ballooning oscillations

$$
\begin{array}{cc}
T(\mathrm{~K})=2.4 \times 10^{-8} \frac{l^{2} \varepsilon_{b}}{N^{2} P_{b}^{2}} & T(\mathrm{~K})=1.2 \times 10^{-8} \frac{\tilde{r}^{2} \varepsilon_{s}}{P_{s}^{2} \chi} \\
n\left(\mathrm{~cm}^{-3}\right)=5.8 \times 10^{-11} \frac{Q_{b} l^{3} \varepsilon_{b}^{7 / 2} \sin ^{2} 2 \theta}{N^{3} P_{b}^{4}} & n\left(\mathrm{~cm}^{-3}\right)=2 \times 10^{-11} \frac{Q_{s} \tilde{r}^{3} \varepsilon_{s}^{7 / 2} \sin ^{2} 2 \theta}{P_{s}^{4} \chi^{3 / 2}} \\
B(\mathrm{G})=6.8 \times 10^{-17} \frac{Q_{b}^{1 / 2} l^{5 / 2} \varepsilon_{b}^{7 / 4} \sin 2 \theta}{N^{5 / 2} P_{b}^{3}} & B(\mathrm{G})=2.9 \times 10^{-17} \frac{Q_{s}^{1 / 2} \tilde{r}^{5 / 2} \varepsilon_{s}^{7 / 4} \sin 2 \theta}{P_{s}^{3} \chi^{5 / 4}}
\end{array}
$$

Table 1.

Here $\chi=10 \varepsilon_{s} / 3+2, \tilde{r}=\lambda_{i} r$.
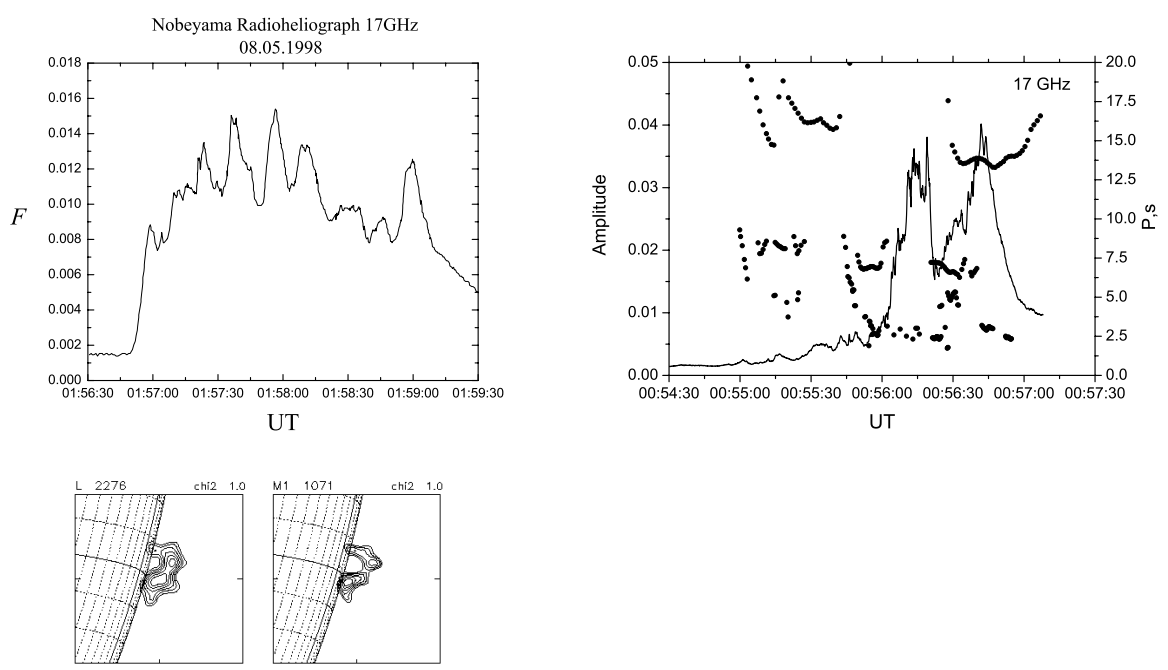

Figure 1. (Left top panel) Time profile of $17 \mathrm{GHz}$ burst from the solar flare of May 8, 1998, obtained with NoRH. Maps of X-ray source in channels L (14-23 keV) and $\mathrm{M}_{1}(22-33 \mathrm{keV})$ obtained onboard the Yohkoh satellite (left bottom panel). (Right panel) Time profile of solar flare of August 28, 1999 at $17 \mathrm{GHz}(\mathrm{NoRH})$, and dynamic spectrum of flux oscillations constructed by means of a wavelet analysis.

of temperature, density, and magnetic field of the flare loop using observation data on the pulsations of microwave emission (Table 1). Time profile of solar flare of May 8, 1998 at microwaves and X-ray maps are presented in Fig. 1(left panels). X-ray source reveals a "tongue-shape" form, which is typical for ballooning disturbances. Pulsations at microwaves can be connected with oscillations of plasma tongues. From Fig. 1 (left panels) it follows that there are four tongues $(N=4)$ in the loop with the length $l \approx 8 \times 10^{9} \mathrm{~cm}$, and $\theta \approx 66^{\circ}$. Fourier analysis of the flare at $17 \mathrm{GHz}$ gives the typical period $P_{b} \approx 16 \mathrm{~s}$. Modulation depth of gyrosynchrotron emission was $\Delta \approx 0.3$, and $Q \approx 25$. From thick target $\mathrm{X}$-ray model the spectral index of electrons was estimated $\delta=4.5$, and thus the plasma beta $\beta=\Delta / \xi \approx 0.11$. Using Table 1 we determine the 
temperature $T \approx 4 \times 10^{7} \mathrm{~K}$, density $n \approx 3.7 \times 10^{10} \mathrm{~cm}^{-3}$, and magnetic field $B \approx 200 \mathrm{G}$ in the flare loop.

NoRH observations of two-loop solar flare of August 28, 1999 have shown that compact $\left(\leqslant 10^{\prime \prime}\right)$ source was located near the sunspot, and the second one $\left(\geqslant 70^{\prime \prime}\right)$ was just above the compact loop. Time profile of radio flare reveals quasi-periodic pulsations (Fig. 1, right panel). Wavelet analysis gives three main branches with the periods of about $14 \mathrm{~s}, 7 \mathrm{~s}$, and $2.4 \mathrm{~s}$. The following scenario can be suggested. The flare energy release is accompanied by the loop-loop coalescence through the development of ballooning instability in the compact loop. As it seen from Fig. 1 (right panel) the oscillations with the period $\approx 14 \mathrm{~s}$, which can be identify with the ballooning mode, has a gap (00:55:45 00:56:30 UT) that coincides with the onset of propagation of the energetic electron front along the extended loop (Yokoyama et al. 2002). It would be natural to attribute this feature to a rise in the gas pressure and to the violation of oscillation conditions in the compact loop, which led to the development of ballooning instability and the injection of hot plasma and energetic particles into the extended loop. As soon as the compact loop was liberated from the excess pressure, the oscillations of plasma tongues resumed. $7 \mathrm{~s}$ oscillations is the second harmonic. Since the oscillations with the period $\approx 2.4 \mathrm{~s}$ emerged only after injection the plasma and energetic particles into the large loop, fast sausage mode is most likely responsible for this oscillations.

Period of ballooning oscillations for the fundamental mode is $P_{b}=l / c_{A}$ where $l \approx$ $\pi \times 10^{\prime \prime} \approx 2 \times 10^{9} \mathrm{~cm}$. From the observation data we find for both modes the Qfactors, $Q_{b}=10, Q_{s}=15$, the modulation depths $\Delta_{b}=0.4, \Delta_{s}=0.1$, and spectral indexes $\delta_{b}=3, \delta_{s}=4$. The radius of extended loop is $r \approx 3 \times 10^{8} \mathrm{~cm}$. Taking for example $\theta \approx 45^{\circ}$ and using Table 1 we obtain the plasma parameters for the extended loop: $T=2.5 \times 10^{7} \mathrm{~K}, n \approx 2.1 \times 10^{10} \mathrm{~cm}^{-3}, B \approx 200 \mathrm{G}$, and for compact one: $T=$ $5.2 \times 10^{7} \mathrm{~K}, n \approx 4.8 \times 10^{10} \mathrm{~cm}^{-3}, B \approx 300 \mathrm{G}$.

\section{Coronal loop as an equivalent electric circuit}

Based on the measurements of Severny (1965) indicating currents $I \approx 3 \times 10^{11} \mathrm{~A}$ in the neighborhood of sunspots Alfven \& Carlqvist (1967) proposed the electric circuit analog of solar flare. Afterwards this idea was applied successfully to the physics of coronal loops (e.g. Spicer 1977; Ionson 1982; Zaitsev \& Stepanov 1992; Zaitsev, Urpo \& Stepanov 2000). As for flare model with the current $I \approx 10^{11}-10^{12} \mathrm{~A}$, the resistance $R \approx 10^{-4}$ $10^{-2} \mathrm{Ohm}$ is required to explain the flare energy release rate $d E / d t=R I^{2} \approx 10^{20} \mathrm{~W}$. The Spitzer resistance under solar condition is too low, $R_{0} \approx 10^{-11} \mathrm{Ohm}$. Zaitsev \& Stepanov (1992) pointed out on two points: (a) flare is essential non-steady-state process and the Ohm's law in the form $\mathbf{j}=\sigma \mathbf{E}$ is non-adequate; (b) dominant role in the electric current dissipation plays the neutral component of flare plasma. In this case the Cowling resistance appears which is 7-9 orders larger compared to the Spitzer one.

The generalized Ohm's law

$$
\mathbf{E}^{*}=\frac{\mathbf{j}}{\sigma}+\frac{\mathbf{j} \times \mathbf{B}}{e n c}-\frac{\nabla p_{e}}{e n}+\frac{F}{c n m_{i} \nu_{i a}}\left[\left(n_{a} m_{a} \mathbf{g}-\nabla p_{a}\right) \times \mathbf{B}\right]-\frac{F^{2}}{c n m_{i} \nu_{i a}} \rho \frac{d \mathbf{V}}{d t} \times \mathbf{B}
$$

together with the Maxwell equations, the equation of motion for the bulk plasma, and the mass conservation law describe self-consistently the behavior of the plasma and the electromagnetic fields. Here $\mathbf{E}^{*}=\mathbf{E}+\mathbf{V} \times \mathbf{B} / c, \rho=n_{a} m_{a}+n_{e} m_{e}+n_{i} m_{i}, p=p_{a}+p_{e}+p_{i}$, $\sigma$ is the Spitzer conductivity, $F=\rho_{a} / \rho$ is the relative density of neutrals. 
The rate of Joule dissipation is

$$
q=\mathbf{E}^{*} \mathbf{j}=\frac{j^{2}}{\sigma}+\frac{F^{2}}{c^{2} n m_{i} \nu_{i a}}(\mathbf{j} \times \mathbf{B})^{2} .
$$

From Eq. (3.2) it follows that in force-free magnetic field $(\mathbf{j} \| \mathbf{B})$ the current dissipation is due to Spitzer conductivity. The dissipation is most powerful in the case $\mathbf{j} \perp \mathbf{B}$. The reason for enhanced current dissipation (flare trigger) can be the flute instability of the chromosphere or prominence. Flute instability yields the penetration of partially-ionized plasma tongues into current channel (Fig. 2) which deform the magnetic field of a loop. As a result the Ampere force $\mathbf{j} \times \mathbf{B}$ appears and enhanced current dissipation is realized. This is because the energy of ions moving through the neutral gas due to Ampere force is much larger than the energy of the relative motion of electrons and ions. Ion-neutral collisions imply great additional dissipation of the energy of electric current.

After integrating Eq. (3.2) over cylindrical volume we find energy release power

$$
\frac{d E}{d t}=\left[\frac{m_{e}\left(\nu_{e i}+\nu_{e a}\right) d}{e^{2} n S}+\frac{2 \pi F^{2} I^{2} d}{c^{4} n m_{i} \nu_{i a} S^{2}}\right] I^{2}=\left[R_{c}+R_{n l}(I)\right] I^{2} .
$$

Taking the scale of tongue of partially-ionized plasma $d \approx 5 \times 10^{7} \mathrm{~cm}$, the loop crosssectional area $S \approx 3 \times 10^{16} \mathrm{~cm}^{2}, I \approx 3 \times 10^{11} \mathrm{~A}, n \approx 10^{11} \mathrm{~cm}^{-3}, T \approx 10^{4} \mathrm{~K}, F \approx 0.5$ from Eq. (3.3) we can find the Cowling resistance $R_{n l} \approx 10^{-3}$ Ohm which supply flare energy release.

For small deviation of the current in a loop we can get the global electrodynamics equation (Zaitsev et al. 1998):

$$
\frac{1}{c^{2}} L \frac{\partial^{2} I_{\sim}}{\partial t^{2}}+R_{n l}(I) \frac{\partial I_{\sim}}{\partial t}+\frac{1}{C(I)} I_{\sim}=0,\left|I_{\sim}\right| \ll I .
$$

Inductance and capacitance is determined mainly by the coronal part of a loop:

$$
L=4 l\left(\ln \frac{8 l}{\pi r}-\frac{7}{4}\right), C(I)=\frac{c^{4} \rho S^{2}}{2 \pi l I^{2}} .
$$

The period of eigen-oscillations of an equivalent RLC-circuit is derived as:

$$
P=\frac{2 \pi}{c} \sqrt{L C(I)} \approx 10 / I_{11} \mathrm{~s}
$$

Here $I_{11}=10^{-11} I$ A and we supposed that $n=10^{10} \mathrm{~cm}^{-3}, l=5 \times 10^{9} \mathrm{~cm}, S=10^{17} \mathrm{~cm}^{2}$. Loop inductance is $L \approx 10^{10} \mathrm{~cm} \approx 10 \mathrm{H}$, so the total energy in the solar coronal loop $L I^{2} / 2 c^{2} \approx 10^{30}-10^{32} \mathrm{erg}$. From the analysis of the Metsähovi flare events at 37 and $22 \mathrm{GHz}$ Zaitsev et al. (1998) concluded that only about $5 \%$ of the energy stored in the loop were released in the course of flares.

RLC-oscillations give periodical variations of loop parameters which in turn produce the modulation of the emission. Analysis of solar flares observed with Metsähovi radio telescope at $37 \mathrm{GHz}$ have been performed by Zaitsev et al. (2001) and Kislyakov et al. (2003) using the Wigner-Ville transform (Cohen 1989). There are two examples.

(i) As it follows from Eq.(3.6) the negative frequency drift in the dynamic spectrum of the flare burst on March 24, 1991 (Fig. 3, left panel) means decreasing of the electric current from $9 \times 10^{11}$ to $10^{11} \mathrm{~A}$, and positive drift indicate the current growth. This is a good illustration of dissipation of the electric current energy during the flare $\left(\approx 10^{28} \mathrm{erg} / \mathrm{s}\right)$ and accumulation of the energy after flare (Zaitsev et al. 2001).

(ii) Fig. 3 (right panel) shows frequency modulation of $37 \mathrm{GHz}$ flux density with average periods of $5 \mathrm{~min}$ in the solar flare of August 28, 1990. Kislyakov et al. (2003) interpreted 


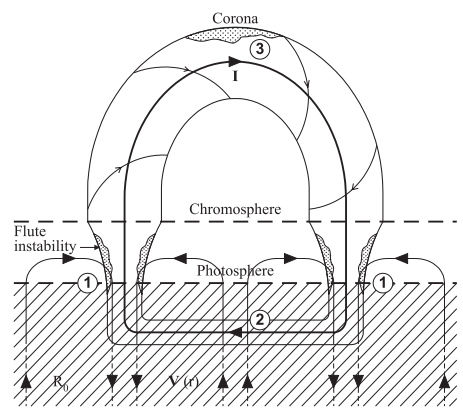

Figure 2. Cartoon model of current-carrying magnetic loop. Convective plasma flows $V_{c} \approx 0.3-1 \mathrm{~km} / \mathrm{s}$ generate current in region 1 . This current flows trough the coronal part of a loop from one foot point to another and closes in region 2 (level $\tau_{5000} \approx 1$ ). Flute instability gives high Cowling resistance in current channel.
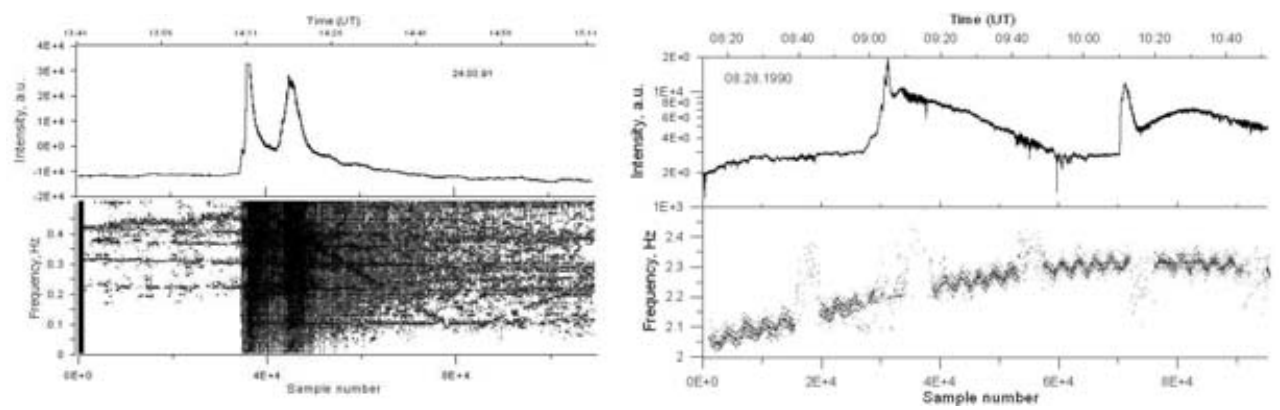

Figure 3. (Left panel) Solar flare burst of March 24, 1991 at $37 \mathrm{GHz}$ (Metsähovi) and dynamic spectrum of low-frequency pulsations obtained using WV-transform. (Right panel) Solar flare burst of August 28, 1990 at $37 \mathrm{GHz}$ (Metsähovi) and dynamic spectrum of low-frequency pulsations.

5 min frequency modulation as a response of the loop to the photosphere oscillations (p-modes). Indeed, coronal loops are connected directly with convection zones because the foot points of a current-carrying loop are closed in the photosphere. This is strong indication on the genetic connection of solar photosphere with the chromosphere and low corona where the sources of microwave emission are located.

Stellar flares also reveals pulsations. Dynamic spectra of LF-pulsations from AD Leo flare of May 19, 1997 observed at 4.85 Ghz with Effelsberg radio telescope displays pulsations with the frequencies $0.5-2.0 \mathrm{~Hz}$. From the WV-analysis Zaitsev et al. (2004) concluded that both the fast sausage mode and RLC-oscillations are presented. Using pulsation characteristics the following parameters of AD Leo flare loop were obtained: Alfven velocity $c_{A} \approx 3.5 \times 10^{8} \mathrm{~cm} / \mathrm{s}$, radius $r \approx 1.8 \times 10^{8} \mathrm{~cm}$, length $l \approx 4 \times 10^{10} \mathrm{~cm}$, current value $I \approx 4.5 \times 10^{12} \mathrm{~A}$, inductance $L \approx 5 \times 10^{10} \mathrm{~cm} \approx 50 \mathrm{H}$, the energy stored in the loop $E=L I^{2} / 2 c^{2} \approx 5 \times 10^{33} \mathrm{erg}$. The rate of energy release was $d E / d t \approx 10^{32} \mathrm{erg} / \mathrm{s}$, which is $2-3$ orders larger than in typical solar flares. This is because AD Leo has stronger magnetic fields and more active surface convection compared to the Sun.

\section{Loop as a magnetic mirror trap}

Among various mechanisms of charged particle acceleration in astrophysics the most effective is the acceleration in DC electric field. From the generalized Ohm's law (Eq. 3.1) 
one can find the parallel component of the electric field (Zaitsev, Urpo \& Stepanov 2000):

$$
E_{\|}=\frac{\mathbf{E B}}{B}=-\frac{\nabla p_{e} \mathbf{B}}{e n B} \approx \frac{1-F}{2-F} \frac{\sigma V_{c} B^{2}}{e^{2} n c\left(1+\psi B^{2}\right)} \frac{B_{r}}{B} .
$$

Here $V_{c} \approx 0.3-1 \mathrm{~km} / \mathrm{s}$ is the horizontal velocity of convective flow on the Sun, $\psi=$ $\sigma F^{2} /(2-F) c^{2} n m_{i} \nu_{i a}$. If the radial component of the magnetic field in a loop $B_{r}=0$ than $E_{\|}=0$ and no acceleration. Flute instability released at quite low $\beta>2 \pi r d / l^{2} \approx 0.01$ and yields the penetration of plasma tongues into the current channel. As the result a radial component of the magnetic field $B_{r}$ appears and electron acceleration becomes possible. An estimations have shown that the number of runaway electrons per second accelerated in DC-electric field of sub-Dricer value $\left(E_{D} / E_{\|} \approx 25\right)$ under solar flare loop condition is $\dot{N}(100 \mathrm{keV}) \approx 10^{35} \mathrm{el} / \mathrm{s}$. Hence penetrating of partially-ionized plasma into current-carrying loop gives Joule heating and particle acceleration with the same time history. This is in favor of the paradigm that heating and acceleration are two aspects of the flare process.

High-energy electrons in a coronal magnetic loop form the distribution "thermal plasma + energetic particle with loss-cone" which are unstable against various modes of electromagnetic and longitudinal waves. Indeed because gyro-radius of collisionless $\sim 100 \mathrm{keV}$ electrons in the loop field $B \sim 100 \mathrm{G}$ is $r_{H} \approx 10 \mathrm{~cm} \ll l$ the particles with the impulse ratio $p_{\perp} / p_{\|}<\left(B_{\max } / B_{\min }\right)^{1 / 2}$ do not trapped in the loop and precipitated at the footpoints. Here $B_{\max } / B_{\min }$ is magnetic mirror ratio. Trapped high-energy electrons have anisotropic distribution. For the applications it is convenient to represent the electron distribution in the form:

$$
f_{\iota} \propto n_{\iota} p_{\perp}^{2 \mu} \exp \left(-p^{2} / \Delta p_{\iota}^{2}\right)
$$

where $\mu=0$ for the loop background plasma $(\iota=0)$ and $\mu=1,2,3$ for the energetic particles $\left(\iota=1, n_{1} \ll n_{0}\right)$.

Loss-cone instability of $\mathrm{x}$-and o-modes near harmonics of electron cyclotron frequency (Electron Cyclotron Maser - ECM) is realized under solar corona condition (Stepanov 1978). Because ECM emission directed almost perpendicular to the magnetic field the problem of the escape emission from the solar and stellar coronae arises due to strong gyro-absorption on hot $\left(10^{7}-10^{8} \mathrm{~K}\right)$ coronal plasma. There is only narrow $\left(5^{\circ}-10^{\circ}\right)$ window along the magnetic field for escaping of the o-mode (Stepanov et al. 1999). Under such conditions the most effective radio emission mechanism can be the plasma radiation mechanism. Upper hybrid (Langmuir) waves are generated first than Langmuir waves convert into electromagnetic ones due to wave-particle and wave-wave interactions. Therewith radiation pattern of the fundamental tone directed mainly along magnetic field and no escape problem arises.

Besides another modes can excite due to loss-cone anisotropy. In particular, Bernstein waves (Zheleznyakov \& Zlotnik 1975) and whistlers (Kuijpers 1975) which are responsible for fine structure of solar radio burst. Whistlers and Alfven waves determine also the dynamics and propagation of electrons and ions in a loop (Bespalov, Zaitsev \& Stepanov 1991). One of the best evidence of wave-particle interaction in solar corona observed by Nobeyama Radioheliograph was the flare event of August 28, 1999 (Yokoyama et al. 2002). In this event relativistic electrons generating gyrosynchrotron emission at $17 \mathrm{GHz}$ propagate along loop axis with the velocity of about $10^{4} \mathrm{~km} / \mathrm{s}$, which is 30 times less than the light velocity. This anomalous propagation can be interpreted in terms of the collective effects of interaction of high-energy particles with small-scale turbulence (Bespalov, Zaitsev \& Stepanov 1991). A cloud of $\sim 1 \mathrm{MeV}$ electrons responsible for microwave emission generates low-frequency whistlers and a turbulent "wall" in the loop is formed. The 
electrons undergo strong resonant scattering and the emission front propagates with the wave phase velocity, which is much lower than particle velocity.

\section{Conclusions}

One of the challenged problems in astrophysics is the origin of the heating of stellar coronae. Therewith it is suggested that the important role in the heating processes play coronal magnetic loops. For example, Nakariakov et al. (1999) supposed that low Q-factor of the loop kink-mode oscillations observed by TRACE is due to anomalous viscosity and resistivity supplying high dissipation of wave energy inside the loop. However they didn't take into account acoustic damping. Loop oscillations lost energy for movement of surrounding plasma (Tsap \& Kopylova 2001).

Recently Zaitsev \& Shibasaki (2004) suggested the current dissipation model for coronal heating which sounds promising. They pointed into attention that most important element for dissipation of diamagnetic currents in solar loops is He I, which has no forbid for de-electron recombination and has larger ionization potential compared to hydrogen.

\section{Acknowledgements}

This work is supported by "Astronomy" Program, the Program of Russian Academy of Sciences "Non-Steady-State Processes in Astronomy", and by RFBR grant 03-02-17218.

\section{References}

Alfven, H. \& Carlqvist, P. 1967 Solar Phys. 1, 120.

Aschwanden, M.J. 2003 NATO Advances Research Workshop, NATO Sci.Ser.II, 22.

Benz, A., Conway J. \& Güdel M. 1998 Astron. Astrophys. 331, 596.

Bespalov, P.A., Zaitsev, V.V. \& Stepanov, A.V. 1991 Astrophys. J. 374, 369.

Cohen, L. 1989 Proc. IEEE 77, 941.

Ionson, J. 1982 Astrophys. J. 254, 318.

Kislyakov, A.G., Stepanov, A.V., Urpo, S. et al. 2003 Preprint HUT-MET-49, Espoo.

Kuijpers, J. 1975 Solar Phys. 44, 173.

Meerson, B.I., Sasorov, P.V. \& Stepanov, A.V. 1978 Solar Phys. 58, 165.

Nakariakov, V.M., Ofman, L., Deluca, E.E. et al. 1999 Science 285, 862.

Roberts, D., Edwin, P.M. \& Benz A.O. 1984 Astrophys. J. 279, 857.

Rosenberg, H. 1970 Astron. Astrophys. 9, 159.

Schrjiver, C.J., Title, A.M., Berger, T.E. et al. 1999 Solar Phys. 187, 261.

Severny, A.B. 1965 Space Sci. Rev., 451.

Spicer, D.F. 1977 Solar Phys. 53, 305.

Stepanov, A.V. 1978 Sov. Astron. Lett. 4, 103.

Stepanov, A.V., Kliem, B., Kruger, A. et al. 1999 Astrophys. J. 524, 961.

Stepanov, A.V., Kopylova, Yu.G., Tsap, Yu.T. et al. 2004 Astron. Lett. 30, 480.

Tsap, Yu.T., Kopylova, Yu.G. 2001 Astron. Lett. 27, 737.

Zaitsev, V.V. \& Stepanov, A.V. 1992 Solar Phys. 139, 343.

Zaitsev, V.V., Stepanov, A.V., Urpo, S. \& Pohjolainen, S. 1998 Astron. Astrophys. 337, 887.

Zaitsev, V.V., Urpo, S. \& Stepanov, A.V. 2000 Astron. Astrophys. 357, 1105.

Zaitsev, V.V., Kislyakov, A.G., Urpo, S. \& Shkelev, V.I. 2001 Radiophysics and Quantum Electronics 44, 697.

Zaitsev, V.V., Kislyakov, A.G., Stepanov, A.V. et al. 2004 Astron. Lett. 30, 319.

Zaitsev, V.V., \& Shibasaki, K. 2004 Astron. Astrophys. (in press).

Zheleznyakov, V.V. \& Zlotnik, E.Ya. 1975 Solar Phys. 43, 431.

Yokoyama, T., Nakajima, H., Shibasaki, K. et al. 2002 Astrophys. J. 576, L87. 